\title{
Techniques Used to Study the Effectiveness of Sintering in Ferrous Powder Metallurgy (P/M) Materials
}

\author{
T. F. Murphy
}

Hoeganaes Corporation, 1001 Taylors Lane, Cinnaminson, NJ 08077, USA

The physical and mechanical properties of pressed and sintered $\mathrm{P} / \mathrm{M}$ materials are developed during the sintering process. These occur through the formation of metallurgical bonds between the base powder particles and diffusion of the added alloying materials. Property enhancements are further realized as the sintering time and/or temperature are increased. The effects of sintering are visible as modifications to features in the microstructure, such as the original particle boundaries and the pore surfaces. Quantification of these features using their geometric characteristics is used as the basis for determining changes in the relative degree-of-sinter. A very small sample of the available literature is shown as references 1-4.

The progression of sintering of an $\mathrm{Fe}-2 \mathrm{Cu}-0.5 \mathrm{C}$ is shown in Figure 1, a - e, where the particle boundaries and unmelted $\mathrm{Cu}$ particles are visible in the green body (1a). As sintering progresses the carbon, from the graphite addition, diffuses (1b) and the $\mathrm{Cu}$ melts (1c) moving, first toward the pore edges and then diffusing along grain and particle boundaries. In Figures $1 \mathrm{~d}$ and e, the $\mathrm{Cu}$ alloying is seen as a dark stain at particle interfaces and grain boundaries. Additionally, the microstructural changes include the lessening or disappearance of the particle boundaries, a smoothing of pore edges, and a reduction in the number of angular features at junctions between particles with increased sintering. It is through the quantification of these features, on properly prepared metallographic specimens, that the effectiveness of sintering is quantified.

Two techniques are described to separate specimens sintered to varying degrees. In Figure 2 , an array of cycloid "waves" is overlaid on an unetched image of a well-sintered material. In practice, a counter is incremented as the cycloids cross a pore or particle boundary. From these counts, and knowing the length of the cycloid array, the surface-tovolume ratio of the pores or particle boundaries to the material volume can be calculated.

Figures 3 and 4 demonstrate the use of a fractal-like measurement, the box count dimension. The outlined rectangle in the upper left portion of Figure 3 is extracted for use in Figure 4, where boxes of various sizes are overlaid onto unetched images. Boxes containing feature segments are counted and a log-log plot relating the counts and the box sizes is made. The box count dimension is the slope of the best-fit linear regression line.

References:

[1] H. F. Fischmeister, Powder Metallurgy International 7(4) (1975) 178.

[2] R. T. DeHoff and F. N. Rhines, Second European Symposium on Powder Metallurgy Stuttgart, Germany, 19681.

[3] J. L. Chermant, M. Coster, J. P. Jernot, Mem.Etud.Sci.Rev.Metall. 1984 81(1) 5.

[4] I. Nettleship et.al., Advances in Powder Metallurgy and Particulate Materials-1997, Metal Powder Industries Federation, Princeton NJ, 1997 16-23. 


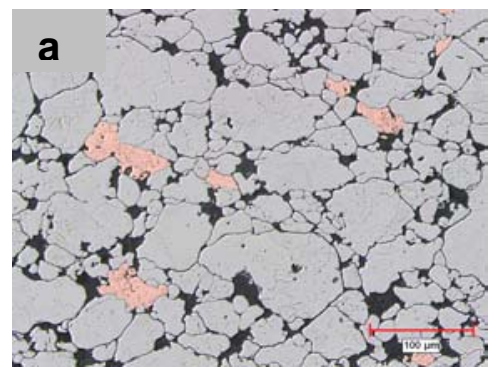

Figure 1. Images showing the progression of sintering: a) "green" sample, b) low temperature sinter, c)melting of the $\mathrm{Cu}, \mathrm{d}$ ) alloying of the $\mathrm{Cu}$ and $\mathrm{C}, \mathrm{e})$ normal sintered microstructure
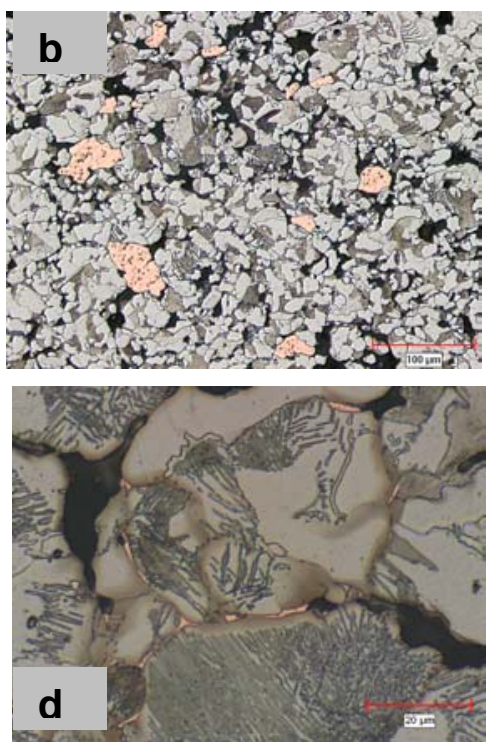
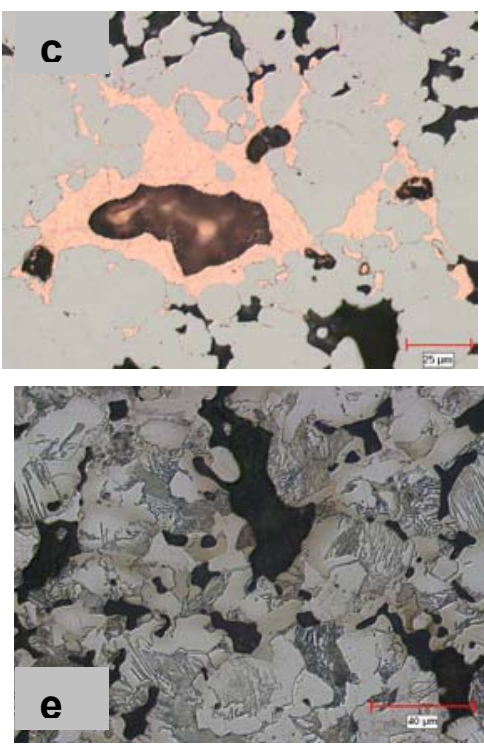

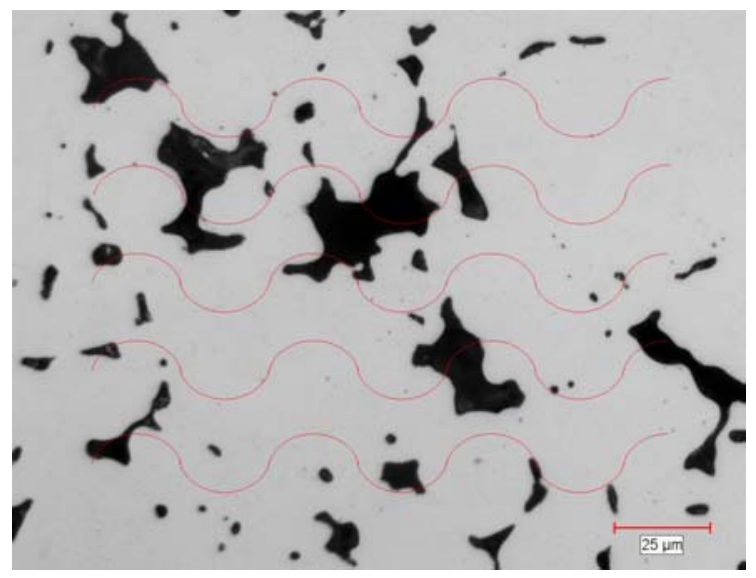

Figure 2. Cycloid array overlaid on an image of a well-sintered material.

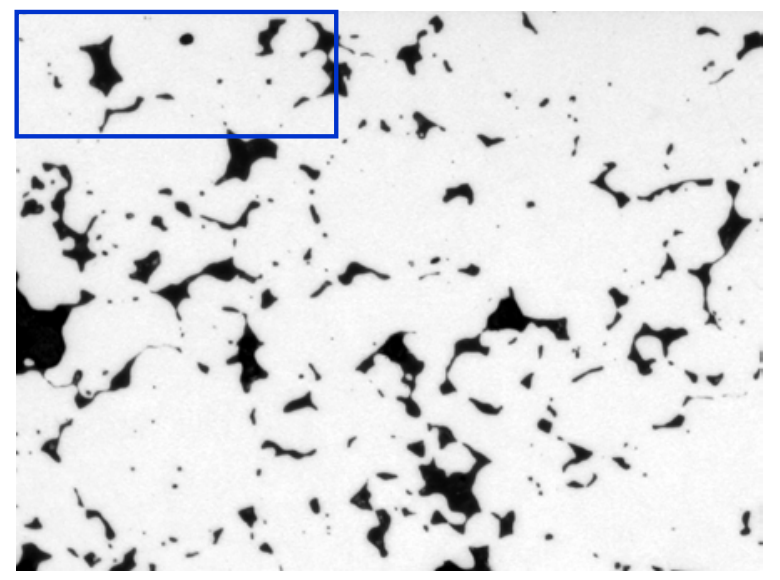

Figure 3. Sample used to illustrate use of the box counting dimension.
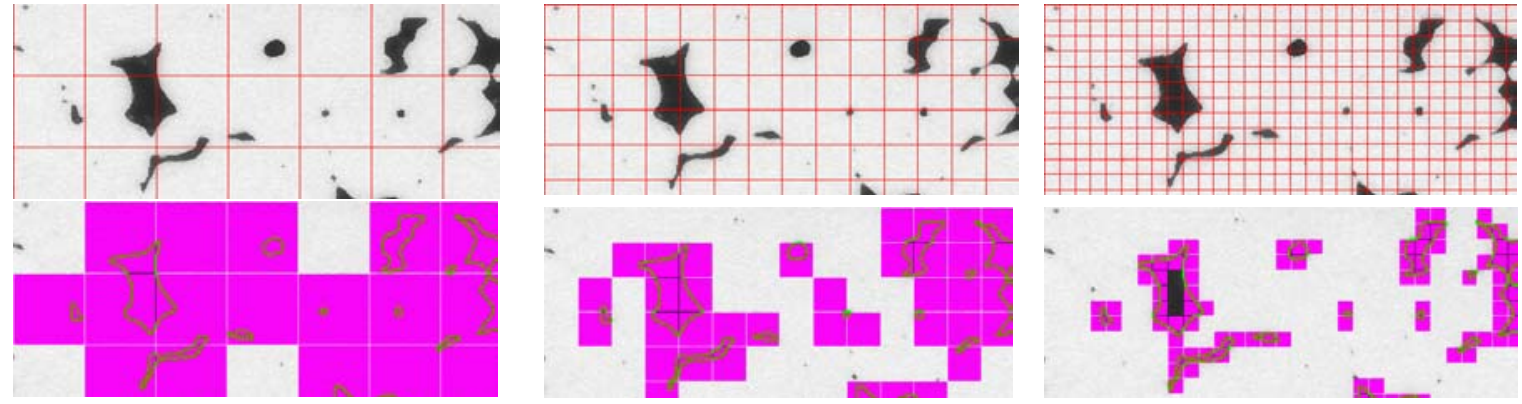

Figure 4. Various size boxes are overlaid onto the image. Boxes containing segments of the features of interest (pore edges) are separated (pink) and counted. A log-log plot is made of the relationship of the number of boxes counted at the corresponding box sizes. The box count dimension is the slope of the linear regression line fitting the points. 